\title{
A importância de ligas acadêmicas no contexto pandêmico da COVID-19: um relato de experiência
}

The importance of academic leagues in the pandemic contexto of COVID-19: na experience report La importância de las ligas académicas en el contexto pandémico del COVID-19: un relato de experiencia

\section{Resumo}

O presente estudo objetiva relatar a experiência vivenciada por acadêmicos de enfermagem acerca da importância das ligas acadêmicas durante a pandemia da COVID-19, buscando analisar qual a percepção em relação às atividades realizadas de forma remota e quais suas implicações. Trata-se de um relato de experiência com abordagem qualitativa, desenvolvido por meio de um formulário eletrônico para coleta de dados e analisado pela teoria de análise de Bardin. Houve retorno com 18 formulários respondidos e destes, foram levantados dados referentes a importância da liga acadêmica no contexto pandêmico. A partir do formulário, 13 alunos consideram os encontros da liga como uma experiência positiva. Também foi observado que a pandemia implicou alguns impasses para a maioria dos ligantes (67\%), visto que estes relataram que sentiram dificuldade em entrar em uma liga, mostrando que a existência da pandemia afetou de modo significativo a entrada de acadêmicos em ligas. $O$ formulário também abordou quais aspectos positivos proporcionados pela liga durante a pandemia e para esta indagação, a maioria dos discentes 
relataram o compartilhamento de conhecimentos, incentivo à pesquisa, participação de eventos online, socialização e produção científica. Portanto, nota-se que apesar da pandemia, a liga desempenha um importante papel na atualidade, mantendo seus objetivos voltados ao ensino, pesquisa e extensão, oferecendo aos discentes novos formatos de ensino no cenário que estamos vivendo.

Palavras-chave: Liga acadêmica; Ensino; COVID-19; Universidade.

\begin{abstract}
This study aims to report the experience of nursing students about the importance of academic leagues during the COVID-19 pandemic, seeking to analyze their perception of activities performed remotely and what their implications are. This is an experience report with a qualitative approach, developed through an electronic form for data collection and analyzed using Bardin's theory of analysis. Eighteen forms were returned and from these, data were collected regarding the importance of the academic league in the pandemic context. From the form, 13 students consider league meetings to be a positive experience. It was also observed that the pandemic implied some impasses for most ligands (67\%), as they reported that they had difficulty joining a league, showing that the existence of the pandemic significantly affected the entry of academics into leagues. The form also addressed the positive aspects provided by the league during the pandemic and for this question, most students reported sharing knowledge, encouraging research, participating in online events, socialization and scientific production. Therefore, it is noted that despite the pandemic, the league plays an important role today, maintaining its goals aimed at teaching, research and extension, offering students new teaching formats in the scenario we are living.
\end{abstract}

Keywords: Academic league; Teaching; COVID-19; University.

\title{
Resumen
}

Este estudio tiene como objetivo reportar la experiencia de estudiantes de enfermería sobre la importancia de las ligas académicas durante la pandemia COVID-19, buscando analizar su percepción de las actividades realizadas de forma remota y cuáles son sus implicaciones. Se trata de un informe de experiencia con enfoque cualitativo, desarrollado a través de un formulario electrónico para la recolección de datos y analizado utilizando la teoría de análisis de Bardin. Se devolvieron dieciocho formularios y de estos se recopilaron datos sobre la importancia de la liga académica en el contexto de la pandemia. Desde el formulario, 13 estudiantes consideran que las reuniones de la liga son una experiencia positiva. También se observó que la pandemia implicó algunos impasses para la mayoría de ligandos (67\%), ya que informaron que tenían dificultad para ingresar a una liga, evidenciando que la existencia de la pandemia afectó significativamente el ingreso de académicos a ligas. El formulario también abordó los aspectos positivos brindados por la liga durante la pandemia y para esta pregunta, la mayoría de los estudiantes reportaron compartir conocimientos, fomentar la investigación, participar en eventos en línea, socialización y producción científica. Por ello, se observa que a pesar de la pandemia, la liga juega hoy un papel importante, manteniendo sus objetivos orientados a la docencia, la investigación y la extensión, ofreciendo a los estudiantes nuevos formatos de enseñanza en el escenario que vivimos.

Palabras clave: Liga académica; Ensenãnza; COVID-19; Universidad.

\section{Introdução}

Levando em consideração aspectos culturais, socioambientais e científicos, a educação superior não está limitada somente no âmbito da formação profissional e sim em fatores subjetivos que envolvem cidadania, interação, disciplina, compromisso e habilidades. Dessa forma, pode-se citar a Liga Acadêmica como uma importante forma e meio extracurricular de aperfeiçoamento de tais componentes que abrangem a formação acadêmica (Silva et al., 2015). Por conseguinte, a área abordada, prática, melhora do currículo e outros benefícios, são fatores que motivam o estudante a participar, tornando cada vez mais viável a criação de diversas Ligas Acadêmicas no país (Georgen, 2017).

No Brasil, a primeira liga acadêmica chamada Liga de Combate a Sífilis foi criada em 1920 na cidade de São Paulo. No entanto, as ligas acadêmicas somente ganharam mais alcance durante o período da Ditadura Militar, no qual os estudantes começaram a questionar mais os métodos e aplicabilidade de ensino - aprendizagem, evidenciando a necessidade de mecanismos que auxiliassem a formação profissional (Filho, 2011).

Sob esse viés, as Ligas Acadêmicas são entidades vinculadas a uma instituição de ensino superior, sem fins lucrativos, formadas por discentes e orientadas por um docente vinculado. Podem ser multiprofissional ou oniprofissional e têm como principal objetivo viabilizar aos participantes vivências que abrangem a integração entre ensino, pesquisa e extensão, proporcionando mais aprendizagem sobre determinada área, bem como autonomia aos estudantes (Silva et al., 2018). 
As principais atividades das ligas acadêmicas envolvem aulas teóricas, capacitações, cursos, palestras, apresentações de trabalhos, dinâmicas, ações de extensão e projetos de pesquisa, possibilitando diversos benefícios aos estudantes, como o maior interesse acerca do processo de ensino e aprendizagem em áreas específicas, um maior contato com a comunidade, experiências, e auxílio na formação acadêmica e futuramente profissional do discente (Bendelaque et al., 2019).

Nesse contexto, as ligas acadêmicas exerceram importante papel no que tange ensino e aprendizagem durante a pandemia pela COVID-19, mesmo com todas as adversidades e obstáculos encontrados, houve a busca por ferramentas e mecanismos que possibilitaram a continuidade das atividades desenvolvidas nesse período, assim como mais oportunidades de ingresso e participação (Silva et al., 2020). Por conseguinte, é significante que estudos acerca da importância das ligas acadêmicas sejam realizados, a fim de evidenciar qual sua relevância em um período de crise e isolamento social.

Sendo assim, o presente estudo tem como principal objetivo relatar a experiência vivenciada por acadêmicos de enfermagem acerca da importância das ligas acadêmicas durante a pandemia da COVID-19, buscando analisar qual a percepção em relação às atividades realizadas de forma remota e quais suas implicações.

\section{Metodologia}

O presente estudo trata-se de um relato de experiência com abordagem qualitativa, acerca da vivência de estudantes de enfermagem que participam de uma Liga Acadêmica durante a pandemia da COVID-19. Nos estudos qualitativos, a interpretação do pesquisador acerca do fenômeno analisado possui grande relevância e, usualmente, essa análise é realizada por meio da observação da coleta de dados (Pereira, et al., 2018, Ludke \& André, 2013). Dessa forma, tal estudo foi desenvolvido por meio de uma coleta de dados, pela análise dos resultados da coleta e, sequencialmente, pela discussão dos resultados. As informações foram coletadas a partir de um formulário eletrônico, produzido por meio da plataforma Google Forms.

Para elaboração do formulário de questões, realizou-se uma reunião online com a participação dos autores do artigo para que fossem definidas quais perguntas seriam inseridas. As perguntas selecionadas para contemplarem o formulário e suas assertivas estão presentes na Tabela 1:

Tabela 1: Perguntas do formulário de pesquisa.

\begin{tabular}{|l|l|}
\hline Pergunta & Assertivas \\
\hline Qual é o seu semestre? & Resposta subjetiva \\
\hline Você tentou por muitas vezes entrar em uma liga acadêmica? & $\begin{array}{l}\text { ( ) Sim } \\
\text { ( ) Não }\end{array}$ \\
\hline $\begin{array}{l}\text { Sentiu mais dificuldade de entrar na liga acadêmica por conta da } \\
\text { COVID-19? }\end{array}$ & $\begin{array}{l}\text { ( ) Sim } \\
\text { ( ) Não }\end{array}$ \\
\hline $\begin{array}{l}\text { Na sua opinião, quais os aspectos positivos proporcionados pela } \\
\text { liga durante a pandemia? }\end{array}$ & Resposta subjetiva \\
\hline $\begin{array}{l}\text { Qual sentimento define a sua participação na liga durante a } \\
\text { pandemia? }\end{array}$ & Resposta subjetiva \\
\hline $\begin{array}{l}\text { Em algum momento durante a pandemia você se sentiu } \\
\text { desmotivado quanto aos estudos? }\end{array}$ & $\begin{array}{l}\text { ( ) Sim } \\
\text { ( ) Não }\end{array}$ \\
\hline $\begin{array}{l}\text { Na sua opinião, os encontros virtuais da liga são uma experiência } \\
\text { positiva? }\end{array}$ & Resposta subjetiva \\
\hline
\end{tabular}

Fonte: Dados da pesquisa (2021).

O questionário eletrônico ficou disponível para receber respostas durante o período de três dias, ocorridos no mês de maio de 2020. A partir da coleta, os dados foram analisados por meio da metodologia de Bardin, pautada nas fases de préanálise, exploração do material e tratamento dos resultados, inferência e interpretação (Mendes \& Miskulin, 2017). 
O momento da pré-análise foi voltado para a organização das informações, visando desenvolver o corpus da pesquisa, que é a junção de todos os dados que necessitam de análise. Desse modo, a ordenação das informações advindas do formulário eletrônico, assim como, a tabulação para análise estatística, foram realizadas por meio do programa Windows Microsoft Office Excel. Sequencialmente, o material coletado foi analisado e descrito, para em seguida elaborar sua discussão baseada em um referencial teórico científico.

A pesquisa do referencial teórico foi desenvolvida frente a busca nas bases de dados científicas Biblioteca Virtual em Saúde - BVS, Scientific Eletronic Library Online (SCIELO), Literatura Latino-Americana e do Caribe em Ciências da Saúde (LILACS) e Google Scholar, com a utilização da combinação de palavras-chaves provenientes dos Descritores em Ciências da Saúde (DeCS), sendo elas: COVID-19, Ligas, Acadêmicos e Universidade.

\section{Resultados}

Os resultados estão expostos em duas categorias temáticas, descritas a seguir.

\section{As motivações dos estudantes para participar de ligas acadêmicas durante a pandemia}

Durante o período de extração de dados, que ocorreu entre os dias 27 a 29 de maio de 2021, mediante formulário dirigido aos integrantes da liga, houve retorno com 18 formulários respondidos. Destes, foram levantados dados referentes a importância da liga acadêmica no contexto pandêmico.

É importante salientar que a pandemia de COVID-19 trouxe impactos em diversas áreas com consequências econômicas, políticas, sociais e na educação. Logo, com o isolamento social tornou-se necessário a utilização de ferramentas online para a realização das atividades educacionais e a criação de novos cenários que permitissem a continuidade dessas atividades.

Gráfico 1 - Distribuição do quantitativo de discentes da liga LaMNeo por semestre.

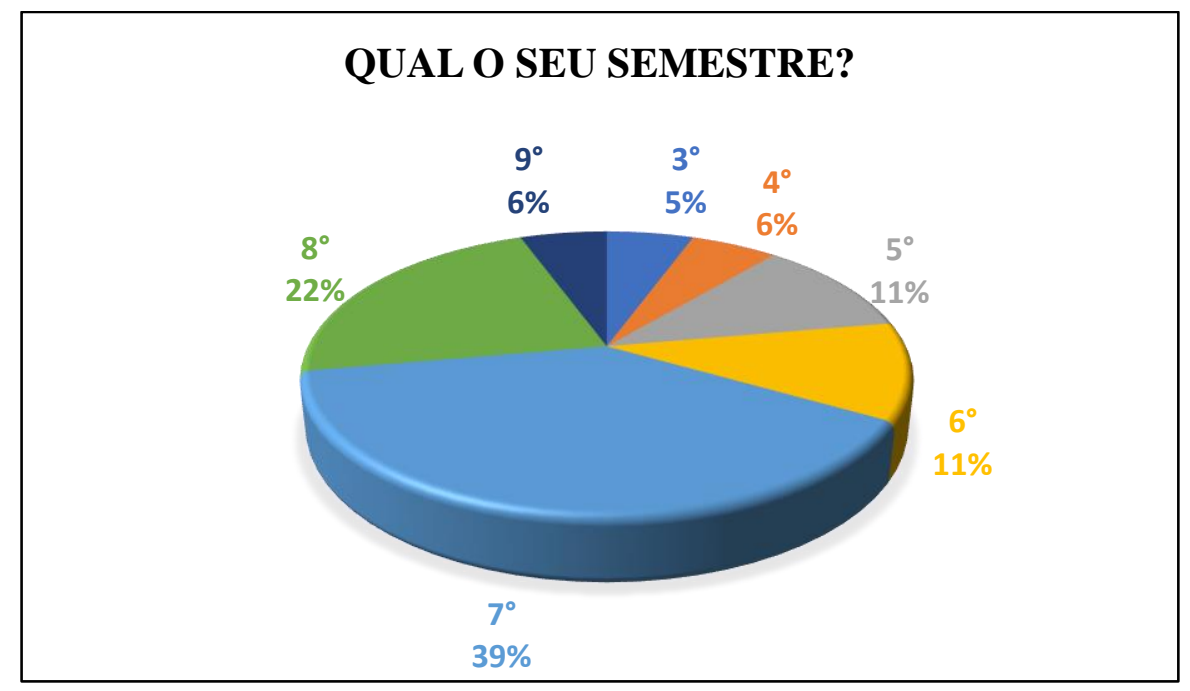

Fonte: Dados da pesquisa.

Com base no levantamento, foi possível identificar que a liga é composta por estudantes do $3^{\circ}$ ao $9^{\circ}$ semestre. Conforme o que consta na Tabela 1, cerca de 39\% do total correspondia a pessoas que estão no $7^{\circ}$ semestre o que representou o maior grupo.

Outro importante dado coletado diz respeito aos aspectos positivos proporcionados pela liga acadêmica durante a pandemia é estar orientado segundo o tripé universitário ensino, pesquisa e extensão. 
Isso pode ser evidenciado nas seguintes falas:

"Compartilhamento de conhecimentos e práticas de forma acessível e dinâmica, mesmo com as adversidades que envolvem a pandemia" (L1).

"Aspectos positivos que a liga acadêmica proporciona e a questão de experiência no tripé do ensino, pesquisa, extensão, ou seja, motiva para estarmos publicando artigos cientificos, dissipando de atividades de extensão" (L16).

Em relação ao sentimento que define a participação dos estudantes na liga acadêmica durante a pandemia, 16 entrevistados relataram sentimentos positivos quanto a sua participação durante a pandemia, dessa forma, percebeu-se alguns pontos relevantes para se entender a percepção dos acadêmicos da graduação em enfermagem na sua participação nesse cenário. Os sentimentos mais frequentes relatados foram gratidão, felicidade e otimismo, demonstrando que apesar de sentimentos negativos também terem sido referidos, existiu um predomínio do relato de sentimentos positivos

Que podem ser observados nas seguintes falas:

"Gratidão, pois a liga acadêmica está me proporcionando grandes experiências para minha formação acadêmica, que irei levar para minha vida profissional (L16)”.

"Sentimento de oportunidade e gratidão por mesmo nesse momento difíicil, poder exercer atividades extra curriculares (L18)".

Em relação aos aspectos positivos proporcionados pela liga durante a pandemia, a maioria dos discentes relataram o compartilhamento de conhecimentos, incentivo à pesquisa, participação de eventos online, socialização, produção científica, novas experiências e aquisição de conhecimento. Assim, podemos considerar que apesar da pandemia a liga desempenha um importante papel no atual momento, mantendo seus objetivos voltados ao ensino, pesquisa e extensão, oferecendo aos discentes novos formatos de ensino no cenário que estamos vivendo.

Quanto ao considerarem os encontros virtuais uma experiência positiva, segundo as respostas obtidas, 13 alunos consideram os encontros uma experiência positiva. Cabendo ressaltar que 2 alunos responderam que essa experiência não pode ser considerada totalmente positiva, pois representa um desafio e não são iguais ao modo presencial, enquanto, 1 aluno não considera uma experiência positiva.

\section{Os desafios enfrentados durante a pandemia de COVID-19}

Gráfico 2: quantidade de tentativas para entrar em uma liga acadêmica.

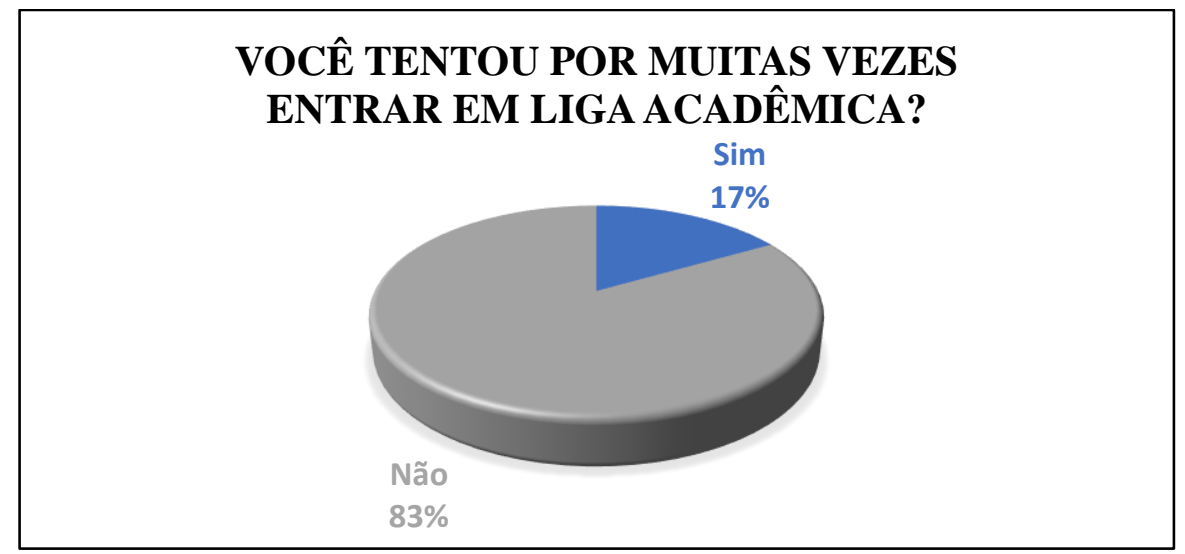

Fonte: Dados da pesquisa. 
Percebeu-se que dos 18 participantes da liga, 17\% tentou mais de uma vez entrar em uma liga acadêmica, enquanto, os $83 \%$ responderam que não. Diante do resultado em questão observa-se que apenas três acadêmicos tentaram entrar diversas vezes em uma liga acadêmica.

Gráfica 3: dificuldades em entrar na liga acadêmica.

\section{SENTIU MAIS DIFICULDADE EM ENTRAR NA LIGA ACADÊMICA POR CONTA DA PANDEMIA DO COVID-19?}

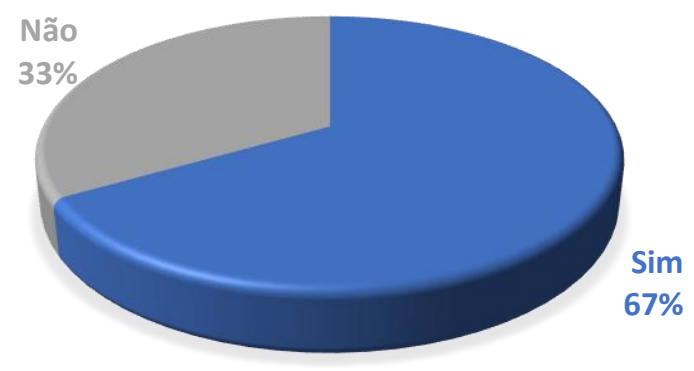

Fonte: Dados da pesquisa.

A quinta pergunta procurou saber se o ligante sentiu mais dificuldade em entrar na liga acadêmica por conta da pandemia do COVID-19. Observa-se que a maioria dos ligantes (67\%) relataram que sim, sentiram dificuldade em entrar em uma liga. Mostrando que a existência da pandemia afetou de modo significativo.

Gráfico 4: desmotivação em relação aos estudos durante a pandemia.

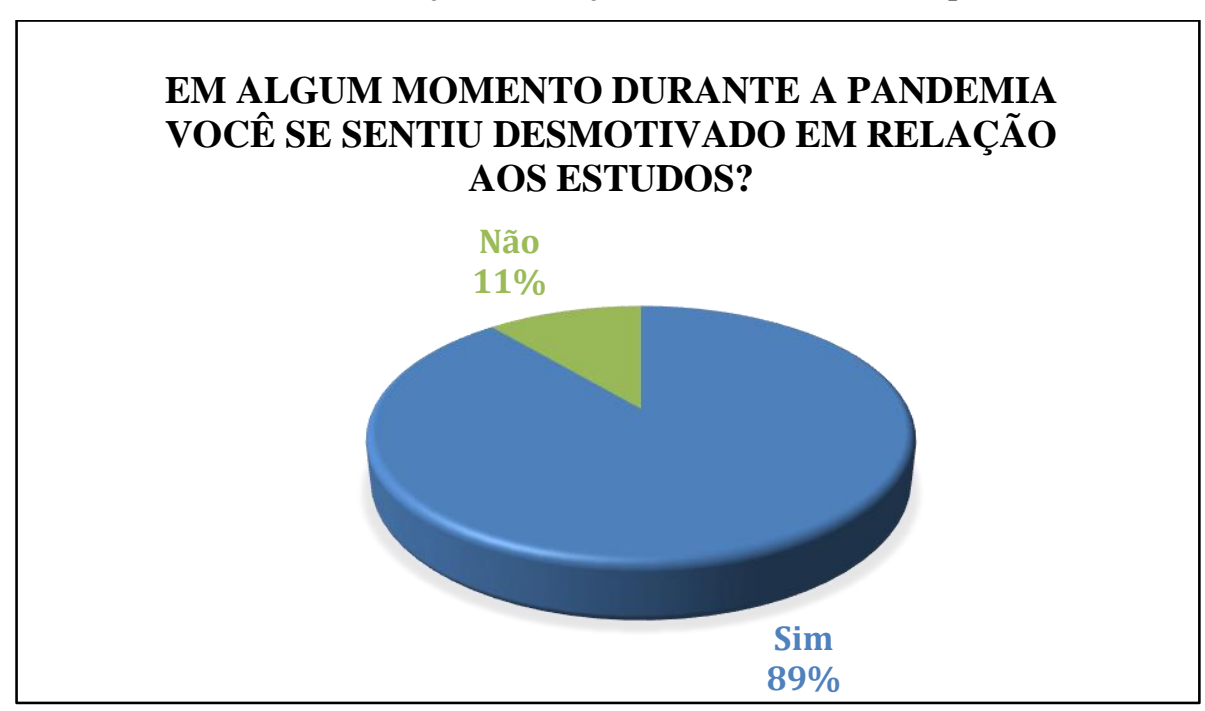

Fonte: Dados da pesquisa.

A última pergunta abordava como um fator desmotivante os estudos dos acadêmicos. Observou-se que a maioria dos discentes responderam que sim (89\%), enquanto, o restante respondeu que não interferiu (11\%). Assim, a inclusão do meio virtual como medida da paralisação das aulas, que se estendeu por mais de um ano, mostra que apesar do intuito de manter a 
oferta da educação os níveis de estresse, depressão e ansiedade tiveram aumento, desde então tem ocorrido um grande desgaste para o seguimento delas, tornando os alunos cada vez mais desmotivados.

\section{Discussão}

Nesse contexto pandêmico, as ligas acadêmicas tiveram que se adaptar ao novo cenário, se organizaram e houve prosseguimento dos encontros via internet em que puderam usar o meio tecnológico para a continuação das aulas (Soares et al., 2020). Para mais, a extensão universitária pode contribuir em novas pesquisas para esse problema sanitário mundial, além de discutir com os membros a importância da liga nesse novo contexto (Oliveira, Andrade \& Lima, 2020). As Ligas Acadêmicas representam as potencialidades para a transformação do cenário de práticas de saúde em variados cenários e pontos da rede de atenção à saúde. Assim, possibilitando a implementação do ensino, pesquisa, extensão na universidade e consolidam a interlocução com a sociedade (Araújo et al., 2019).

Com o distanciamento social, novas formas de acompanhar e assistir as reuniões e aulas das ligas foram incorporadas e iniciou a utilização das plataformas virtuais como meio de interação e aprendizagem (Bessa et al., 2021). Além disso, vale ressaltar que as ligas acadêmicas promovem ações de ensino, pesquisa e extensão - que é o tripé universitário - por isso, a utilização das novas plataformas de ensino proporcionou um aprendizado de novos assuntos assim como não prejudicou o ensino para os alunos ligantes (Silva et al., 2020, Oliveira et al., 2021).

Referente a este aspecto, as ligas acadêmicas tiveram que recriar novos modelos de aprendizagem, utilizando ferramentas para estimular os ligantes no âmbito educacional, como a ampliação de cursos virtuais com certificação de carga horária, gamificação com recursos de tecnologia educacional como o "kahoot", webconferências, além de incentivar os ligantes à produzirem artigos científicos. Logo, a partir do cenário pandêmico, houve a necessidade de buscar novas estratégias para alcançar a articulação entre ensino, pesquisa e extensão (Oliveira et al., 2020).

Além disso, a partir dos discursos dos estudantes é possível apreender que a participação na liga proporcionou sentimentos positivos aos discentes, embora, em cenários pandêmicos como esse no presente momento, os sentimentos de medo, estresse, ansiedade, luto, culpa, raiva e o desamparo diante da incerteza são respostas emocionais comuns. Esses sentimentos juntam-se ainda ao sofrimento relacionado às perdas financeiras, aos processos de discriminação/estigmatização das pessoas acometidas pela doença, o distanciamento social e ruptura das atividades cotidianas (Teixeira \& Dahl, 2020).

Sob essa égide, a ruptura abrupta das atividades presenciais durante a pandemia da covid-19 desencadeou um cenário desafiador para a realização de atividades de ensino-aprendizagem, possibilitando o desenvolvimento de competências e habilidades para romper os desafios presente no contexto atual, e contribuir de forma positiva para a propagação do conhecimento aos alunos e a atenuação dos impactos negativos na esfera acadêmica (Ferreira et al., 2020).

Diante disso, as ligas acadêmicas precisaram se adequar ativamente para a promoção do ensino, mesmo com as atividades presenciais suspensas foi possibilitado um acesso facilitado aos eventos, por meio de plataformas de transmissão online. O meio virtual possibilitou a integração de professores e alunos de várias localidades, sem precisar de grandes adequações logísticas, o que corroborou para novas oportunidades e inovações na educação (Malta et al., 2021).

De acordo com as literaturas encontradas, somado aos resultados apresentados neste trabalho, pode-se afirmar que as ligas acadêmicas são de relevância para a formação dos alunos e, consequentemente, maior incentivo no desenvolvimento na universidade (Camilo, et al, 2020). Além disso, a participação em ligas proporciona aproximação com práticas de ensino, busca de reconhecimento social, ampliação da visão crítica, oferecimento de oportunidades no ensino-aprendizagem e otimizam a educação entre professores e alunos (Araújo, et al., 2019, Yang, et al, 2019).

Ademais, as ligas acadêmicas produziram artigos, trabalhos para apresentação, aulas abertas, reuniões, fizeram planejamento de eventos, e outras atividades que incentivam o desenvolvimento dos discentes na universidade (Soares et al., 
2020). Outrossim, as ligas continuam sendo importantes para os alunos em formação e para a sociedade como um todo, pois colaboram em propagar informações e conhecimentos para a comunidade (Oliveira et al., 2020).

\section{Conclusão}

A chegada da pandemia causada pela Covid-19 trouxe consigo inúmeros impactos que atingiram negativamente o âmbito educacional das universidades. Dentre esses impactos destacam-se o afastamento dos alunos das atividades presenciais, a falha no desenvolvimento de novas metodologias que se adequassem ao contexto pandêmico, além do surgimento de sentimentos prejudiciais para saúde mental por parte dos estudantes, que compõe um grande grupo afetado por essas intempéries.

Dessa forma, a partir dos argumentos supracitados no decorrer do estudo, as ligas acadêmicas mostram-se como boas estratégias para manter e estimular a produtividade dos acadêmicos, contribuindo para a produção de estudos, oferta de cursos e propagação de informação para a sociedade.

Por fim, considerando a escassez de conteúdo nas bases de pesquisas científicas acerca da contribuição de ligas acadêmicas durante a pandemia, pretende-se que este artigo incentive a produção de novos estudos a respeito dessa importante temática. Sugere-se que as próximas pesquisas utilizem uma maior amostragem de acadêmicos para a coleta de dados, visto que assim haverá mais informações para enriquecer o conteúdo do trabalho e será alcançada uma maior exatidão acerca da realidade vivenciada nesse contexto.

\section{Referências}

Araújo, C. R. C, Lopes, R. E, Dias, M. S. A, Neto, F. R. G. X, Farias, Q. L. T, Cavalcante, A. S. T. (2019). Contribuição das ligas acadêmicas para formação em enfermagem. Enfermagem em Foco, 10 (6): 137-142.

Bendelaque, D. F. R, Carvalho, D. N. R, Contente, R. T. C, Cunha, C. S, Ferreira, W. N. A, Lopes, M. F. C, Sousa, I, M. (2019). Contribuição da liga acadêmica na formação e produção de conhecimento em saúde do idoso. Brasilian jornal of health revie, 2 (4), $3762-3773$.

Bessa, A. M, Silva, M. A, Barbosa, T. C. P, Moreira, R. C, Costa, K. P, Santos, R. C, Souza, D. A. S, Carvalho, N. M. (2021). Liga acadêmica de urgência e emergência e suas mídias sociais como estratégia de educação em saúde em tempos de COVID-19. Brazilian Journal of Health Review, 4 (3), $2595-6825$.

Camilo, B. C, Bastos, M. G, Toledo, G. C, Ferreira, A. P, Brandão, T. G, Reis, A. F. M, Coutinho, I. A. P. B, Aranha, G. L, Souza, B. I. A. (2020) Análise das ligas acadêmicas de medicina sob a perspectiva dos alunos. Sciencia Medica, 30, 1-7.

F, A, Príncipe, F, Pereira, H, Oliveira, I, Mota, L. (2020). Covimpact: pandemia covid-19 nos estudantes do ensino superior da saúde. Revista de Investigação \& Inovação em Saúde, 3(1), 7-16.

Filho, P. T. H. (2011). Ligas acadêmicas: motivações e críticas a propósito de um repensar necessário. Revista Brasileira de Educação Médica, 35 (4).

Georgen, D.I. (2017). Ligas Acadêmicas: uma revisão de várias experiências. Arquivos Catarinenses de Medicina, 46(3), $183-193$.

Ludke, M, Andre, M.E. D. A. (2013) Pesquisas em educação: uma abordagem qualitativa. E.P.U.

Malta, C. P, Santos, W. A, Oliveira, P. G. A, Leite, L. P, Soares, A. A. D, Lima, B. A, Vieira, K. S. T, Pena, K. S, Silva, M. E. M, Marcelo Imbroinise Bittencourt, M. I. (2021). Atuação da liga acadêmica de cardiologia durante a pandemia da Covid-19 e as implicações na educação médica. Electronic Journal Collection Health, 2178-2091.

Mendes, R. M. \& Miskulin, R. G. S. (2017). A análise de conteúdo como uma metodologia. Cadernos de pesquisa, 47(165), 1044-1066.

Oliveira, E. N, Andrade, C. S. G, Lima, L. M. C. (2020). A liga interdisciplinar em saúde mental e suas contribuições em tempos de covid-19. Revista Brasileira e Psicologia e educação, 22(2), 2594-8385.

Oliveira, H. T, Garcia, D. R., Jesus, P. C, Santos R. D. S, FANGEL, L. M. G. (2021) Estratégias de continuidade da LATOHCP frente à pandemia da Covid19. Revista Participação, (35), 88-96.

Pereira, A. S, Shitsuka, D. M, Parreira, F. J, Shitsuka, R. (2018) Metodologia da Pesquisa Científica. UFMS. 
Research, Society and Development, v. 11, n. 3, e7611325872, 2022

(CC BY 4.0) | ISSN 2525-3409 | DOI: http://dx.doi.org/10.33448/rsd-v11i3.25872

Rezende, C. A, Silva, M. A, Barbosa, T. C. P, Costa, C. M, Freitas, L. C. D, Santos, I. T, Andrade, S. N, Oliveira, M. M, Santos, R. C. (2021) A importância da liga acadêmica de saúde materno-infantil em tempos de Covid-19: Relato de experiência. International Journal of Development Research, 11 (2), 44721 44723 .

Silva, D.P, Raimundo, A.C.L, Santos, I. M. R, Gomes, N. M. C, Melo, P.D.C.R, Santos, D.S. (2018). Proposição, fundação, implantação e consolidação de uma liga acadêmica. Revista de Enfermagem UFPE, 12 (5), 1486-1492.

Silva, J. H. S, Chiochetta, L. G, Oliveira, L. F. T, Sousa, V. O. (2015). Implementação de uma Liga Acadêmica de Anatomia: desafios e conquistas. Revista Brasileira de Educação Médica, 39 (2), 310- 315.

Silva, W. B. H, Cortes, E. M. P, Marta, C. B, Francisco, M. T. R, Silva, P. O, Santos, R. M, Ferreira, M.A, Neves, M.P, Lima, T. A, Machado, P. R. F. (2020). Reinvenção das ligas acadêmicas em período de pandemia e interrupção das aulas presenciais. Global Academic Nursing Journal, 1 (3).

Soares, I. F, Carvalho, J. M. S, Teive, V. L. S. (2020). O uso de atividades a distância e remotas em uma liga acadêmica universitária: Relato de experiência. Associação Universidade em Rede.

Teixeira MR., Dahl CM. Recriando cotidianos possíveis: construção de estratégias de apoio entre docentes e estudantes de graduação em terapia ocupacional em tempos de pandemia. Rev. Interinst. Bras. Ter. Ocup. 2020..4(3): 509-518.

Yang, G. Y. H, Braga, A. C. B, Hipólito, N. C, Vieira, K. S. T, Pessanha, C. G, Abrantes, A. G, Pereira, P. S, Corrêa, C. L. (2020). Liga de Anatomia Aplicada (LAA): as Múltiplas Perspectivas sobre Participar de uma Liga Acadêmica. Revista Brasileira de Educação Médica. São Paulo, 43 (1). 